\title{
Criminologie
}

\section{Étude de certains déterminants des incendies volontaires à Montréal}

\section{Luc Vallée et Stéphane Dupuis}

Volume 30, numéro 1, printemps 1997

Criminalités économiques

URI : https://id.erudit.org/iderudit/017398ar

DOI : https://doi.org/10.7202/017398ar

Aller au sommaire du numéro

\section{Éditeur(s)}

Les Presses de l'Université de Montréal

ISSN

0316-0041 (imprimé)

1492-1367 (numérique)

Découvrir la revue

Citer cet article

Vallée, L. \& Dupuis, S. (1997). Étude de certains déterminants des incendies volontaires à Montréal. Criminologie, 30(1), 73-92.

https://doi.org/10.7202/017398ar
Résumé de l'article

Research into the determining factors in arson cases has traditionally focused on factors linked to the characteristics of the burned building. One of our basic hypotheses is that deliberately set fires also have an underlying economic motivation.

In this case, the present study confirms the hypothesis that there appears to be an indisputable link between the unemployment rate and mortgage burdens and arson rates, regardless of the phase of the economic cycle in which the arson occurs.

Moreover, the study corroborates the idea that increased surveillance is necessary in areas presenting a higher risk of fraud and having a specific socioeconomic and financial profile. A lower incidence of arson and the improvement of insurers ' ability to predict losses due to arson could lead to a significant reduction in the number of claims, and consequently, in the amount of premiums.

By looking more specifically at the economic motivations influencing arson throughout the different phases of the economic cycle, this study evokes the establisment of a forecasting system that would allow insurance companies to identify the areas of Montreal that present a higher risk level for arson, thus allowing them to establish their rates in a more equitable manner.
Ce document est protégé par la loi sur le droit d'auteur. L'utilisation des services d’Érudit (y compris la reproduction) est assujettie à sa politique d'utilisation que vous pouvez consulter en ligne.

https://apropos.erudit.org/fr/usagers/politique-dutilisation/ 


\section{ÉTUDE DE CERTAINS DÉTERMINANTS DES INCENDIES VOLONTAIRES À MONTRÉAL'}

Luc Vallée ${ }^{2}$

Stéphane Dupuis ${ }^{3}$

Research into the determining factors in arson cases has traditionally focused on factors linked to the characteristics of the burned building. One of our basic hypotheses is that deliberately set fires also have an underlying economic motivation.

In this case, the present study confirms the hypothesis that there appears to be an indisputable link between the unemployment rate and mortgage burdens and arson rates, regardless of the phase of the economic cycle in which the arson occurs.

Moreover, the study corroborates the idea that increased surveillance is necessary in areas presenting a higher risk of fraud and having a specific socioeconomic and financial profile. A lower incidence of arson and the improvement of insurers' ability to predict losses due to arson could lead to a significant reduction in the number of claims, and consequently, in the amount of premiums.

By looking more specifically at the economic motivations influencing arson throughout the different phases of the economic cycle, this study evokes the establisment of a forecasting system that would allow insurance companies to identify the areas of Montreal that present a higher risk level for arson, thus allowing them to establish their rates in a more equitable manner.

\section{INTRODUCTION}

Hormis les incendies qui sont le fait d'enfants, dont le caractère volontaire n'est pas «pur », les incendies volontaires sont classés en trois grands types, soit ceux qui sont le fait : 1) de personnes souffrant de troubles psychiques ; 2) de personnes agissant pour des motifs politiques ; 3 ) de personnes ayant des intentions criminelles particulières (vengeance, mafia,

1. Nous tenons à remercier le Bureau d'assurance du CANADA et l'École des H.E.C. pour leur contribution financière. De même. nous remercions les intervenants des compagnies d'assurances qui ont bien voulu discuter de la question de l'incendie criminel et nous fournir des informations hautement utiles à la recherche.

2. Professeur agrégé aux HEC.

3. M.Sc. Économie. 
fraude à l'assurance, gentrification, etc.). À cet égard, lors de la souscription d'assurances-incendie, les compagnies d'assurances considèrent l'existence, dans l'évaluation des risques d'incendie volontaire (pour des actifs immeubles assurables), de critères objectifs et subjectifs.

Les critères objectifs renvoient à des caractéristiques des actifs concernés (immeuble isolé ou sans surveillance, fréquenté ou non par des enfants et des adolescents, où les mesures de sécurité sont déficientes ou absentes, etc.). L'importance des facteurs dits objectifs apparait dans la plupart des sinistres d'origine criminelle, mais ils ne présentent qu'un intérêt limité quand il s'agit de savoir si un bien est susceptible de faire l'objet d'un incendie volontaire provoqué par des familiers des lieux. Ce sont les critères dits « subjectifs" qui permettent de déterminer si l'assuré risque de mettre le feu à son propre bien. Quand un changement dans la conjoncture menace la rentabilité et la solvabilité d'entreprises de secteurs donnés, il est clair que les facteurs dits objectifs ne sont pas déterminants pour juger des risques d'incendie volontaire (Munich Re, 1992).

Ainsi, dans la tarification de l'assurance-incendie-habitation, les compagnies d'assurances fixent le montant des primes exigées en fonction de critères assez semblables. Premièrement, elles établissent le montant d'une prime de base essentiellement en tenant compte de la protection contre l'incendie, du nombre de logis de l'immeuble, de son âge, du genre de chauffage, du secteur où est situé l'immeuble (urbain, semi-urbain, rural), des ratios coûts des sinistres/primes pour le secteur du risque assuré (expérience acquise) et enfin de la concurrence sur le marché. Elles ajustent ensuite le montant de la prime spécifiquement au risque assuré par la prise en compte d'autres facteurs, qui sont, en général, l'âge de l'assuré, la présence de fumeurs dans le logement, le nombre d'années sans sinistre pour le risque à assurer, la présence d'un système d'alarme relié au poste de pompiers et enfin l'occupation professionnelle de l'assuré.

Les assureurs semblent ainsi se baser surtout sur des critères objectifs liés aux caractéristiques du bâtiment ainsi que sur des critères mixtes (par exemple le ratio sinistres/primes ou l'occupation de l'assuré), ne tenant compte qu'indirectement de critères objectifs sous-jacents de nature plus socio-économique tels le taux de chômage, les charges hypothécaires moyennes, etc.

Dans cet ordre d'idées, plusieurs études ont traité des déterminants de l'incendie criminel, mais relativement peu se sont penchées sur ses fondements socio-économiques. On peut citer, par exemple, Hershbarger et Miller (1978), lesquels établissent que l'incendie criminel à motif économique dépend essentiellement de six facteurs (soit les faillites commerciales ou 
personnelles, le besoin perçu d'éliminer la concurrence, les opérations immobilières dans les quartiers défavorisés, le taux de vacance des immeubles, les conflits de travail et enfin le désir des criminels de cacher leurs crimes). Citons encore Cloninger (1978), qui démontre que l'incendie criminel constitue souvent la façon optimale de se défaire d'une propriété, compte tenu des rendements et des risques et que les pertes monétaires dues aux incendies peuvent être prédites par une étude des caractéristiques des populations concernées.

Cela dit, dans la plupart des cas, l'étude des déterminants des incendies volontaires s'est traditionnellement intéressée aux facteurs liés aux caractéristiques de l'immeuble incendié. L'une de nos hypothèses de base étant que les incendies volontaires ont, si l'on excepte les cas de maladie mentale, de vengeance ou de camouflage d'un crime, un substrat économique, il nous apparaît pertinent de chercher tant du côté des facteurs à caractère socioéconomique que des facteurs liés aux caractéristiques des immeubles pour en expliquer la fréquence des incendies dans des secteurs urbains donnés.

La présente étude cherche donc à établir des liens statistiques entre la probabilité d'incendies et des variables socio-économiques plus ou moins négligées par les compagnies d'assurances, et ce, dans un modèle adapté à la ville de Montréal. Nous tenterons de démontrer l'intérêt de ces liens pour l'établissement des primes d'assurances. Enfin, nous nous proposons de dégager des déterminants socio-économiques dont la stabilité dans le temps, indépendamment de la conjoncture économique, puisse être reconnue.

Par la prise en compte plus précise des motivations économiques de l'incendie criminel à travers les différentes phases du cycle économique, cette étude se propose de contribuer à l'élaboration d'un instrument économétrique de prévision des taux d'incendie, ce qui permettrait d'améliorer et de raffiner l'évaluation et la tarification des risques assurés par les compagnies d'assurances.

Pour commencer, nous faisons un tour $\mathrm{d}$ 'horizon général du contexte montréalais des dernières années en matière d'incendies criminels. Nous décrivons ensuite les données et les variables que nous utilisons. En l'occurrence, les données utilisées se rapportent strictement aux années 1986 et 1991 , et ce pour des raisons de disponibilité des données au niveau des secteurs de recensement. Nous présentons et commentons par la suite les résultats de nos analyses, puis nous terminons avec les conclusions générales à tirer de ces résultats et les recommandations qu'ils peuvent inspirer quant à l'approche générale que pourraient adopter les compagnies d'assurances pour traiter des incendies criminels. 


\section{LES INCENDIES CRIMINELS À MONTRÉAL}

\subsection{Description des données}

Contrairement à d'autres crimes manifestes (cambriolage, etc.), l'incendie criminel est long et difficile à résoudre. Une enquête spécialisée doit en démontrer la nature criminelle et les preuves matérielles sont souvent détruites, ce qui pose des problèmes de fiabilité des données (Généreux, 1987). À Montréal, cette tâche incombe au Service des Incendies, au Service de police de la Communauté urbaine de Montréal (section spécialisée en enquêtes d'incendies criminels) et au Service anti-crime des assureurs (SACA), qui se spécialise dans l'enquête pour fraudes à l'assurance.

Ces multiples sources d'information en renforcent grandement la fiabilité. La banque de données utilisée ici est une compilation de tous les dossiers de la section des enquêtes sur l'incendie criminel du SPCUM pour les années 1986 et 1991. Elle comprend l'ensemble des feux de toutes natures rapportés à la police et survenus sur le territoire montréalais au cours de ces deux années. En outre, la banque de données de la SACA corrige les lacunes de la banque de données du SPCUM.

Un problème fréquent dans l'étude de l'incendie tient à sa classification selon sa nature, qui peut être soit criminelle (donc volontaire), soit accidentelle (non-intentionnelle, par négligence ou suite à une défectuosité), soit indéterminée (dont la cause n'est pas clairement criminelle ou accidentelle) soit encore naturelle (plus rare : combustion spontanée, foudre ou autre). La catégorie « indéterminée » pose problème : on estime en général que $50 \%$ des incendies ainsi classés sont en fait criminels (section des incendies criminels de la SPCUM ; Généreux, 1987). Parce qu'il est reconnu que les incendies dits accidentels sont souvent des incendies criminels (aucun indice ne permettant cependant de les classer comme étant criminels), nous avons trouvé justifié de distribuer également dans les catégories "incendie criminel » et « incendie accidentel » les incendies classés dans la catégorie « indéterminé », et ce pour toutes nos analyses. Par ailleurs, les experts estiment négligeable le nombre d'incendies non signalés, compte tenu de la visibilité du phénomène dans un centre urbain comme Montréal.

Nos données sur les incendies par districts policiers sont ajustées afin de correspondre aux subdivisions du recensement. Les données sur les incendies par districts policiers et les données socio-économiques des diverses subdivisions du recensement sont ainsi combinées avec le nombre d'immeubles des divers secteurs postaux, de sorte que les tests économétriques des modèles soient effectués transversalement en fonction des secteurs postaux. En l'occurrence, les données socio-économiques relatives aux variables indépendantes sont tirées de Statistique Canada, les secteurs du 
recensement ayant été ajustés pour s'adapter aux données obtenues par secteurs postaux. De même, les taux d'incendies criminels par secteurs de recensement sont ajustés en fonction des données de variables indépendantes obtenues par secteurs postaux.

\subsection{Un tour d'horizon des statistiques}

Tableau 1

Statistiques de l'incendie à Montréal de 1982 à 1993

\begin{tabular}{cccc}
\hline Année & $\begin{array}{c}\text { Nombre } \\
\text { d'événements }\end{array}$ & $\begin{array}{c}\text { Nature } \\
\text { accidentelle }\end{array}$ & $\begin{array}{c}\text { Nature } \\
\text { criminelle }\end{array}$ \\
\hline 1982 & 1798 & $791(44 \%)$ & $1007(56 \%)$ \\
1983 & 1433 & $543(38 \%)$ & $890(62 \%)$ \\
1984 & 1430 & $489(34 \%)$ & $941(66 \%)$ \\
1985 & 1372 & $434(32 \%)$ & $938(68 \%)$ \\
1986 & 1299 & $610(47 \%)$ & $689(53 \%)$ \\
1987 & 1463 & $653(45 \%)$ & $810(55 \%)$ \\
1988 & 1290 & $620(48 \%)$ & $670(52 \%)$ \\
1989 & 1589 & $740(47 \%)$ & $849(53 \%)$ \\
1990 & 1963 & $959(49 \%)$ & $1004(51 \%)$ \\
1991 & 1983 & $885(45 \%)$ & $1098(55 \%)$ \\
1992 & 2059 & $823(40 \%)$ & $1236(60 \%)$ \\
1993 & 1784 & $827(46 \%)$ & $957(54 \%)$ \\
\hline Moyenne & 1733 & $787(46 \%)$ & $946(54 \%)$ \\
\hline
\end{tabular}

Source : Terminologie pertinente au secteur de l'incendie volontaire, Ministère des Affaires municipales, Gouvernement du Québec.

N.B. : Sur l'ensemble des événements faisant l'objet d'une enquête, le service des incendies criminels du SPCUM est responsable de l'enquête de $65 \%$ à $70 \%$ de ceux-ci. Les autres $30 \%$ à $35 \%$ sont référés aux districts policiers concernés. Par ailleurs, la classe « indéterminée » est éliminée et les incendies sont traités soit comme criminels, sont comme accidentels (50\%-50\%).

Les données sur l'incendie à Montréal depuis 1982 révèlent d'intéressantes tendances : une baisse des événements de 1982 (sommet des années 1980) à 1986 et une reprise de 1988 à 1992 (1991 constituant un sommet en ce qui concerne les incendies criminels). En 1993, on observait une baisse se situant entre $25 \%$ et $30 \%$, analogue à celle observée de 1982 à 1983 et imputable à la fin de la récession. En moyenne, selon les experts, 57,4\% des incendies sont d'origine criminelle, en tenant compte des incendies dits « indêterminés » et en excluant la proportion inconnue d'incendies « accidentels » qui sont en fait des incendies volontaires (le contraire est plus rare, la preuve d'un incendie criminel étant beaucoup plus consistante que celle d'un iлcendie accidentel). L'estimation des experts est prudente : les incendies criminels classés à tort dans les autres catégories peuvent être largement sous-estimés. 
La fréquence annuelle des incendies rapportés à Montréal (tableau 1) suit sensiblement le cycle économique de cette dernière décennie et témoigne de la plus grande prévalence de l'incendie et du crime en général lors de périodes économiques difficiles. Très élevée en 1982, la tendance décrô̂t jusqu'en 1986 pour, à partir de 1988, faire une remontée fulgurante jusqu'en 1992. Ainsi, alors que la proportion des incendies accidentels augmente durant les années de récession, la proportion des incendies criminels, pour la même période, présente de plus faibles taux et n'augmente que suite aux années de récession. Cela pourrait s'expliquer par une plus grande propension à provoquer des incendies « arrangés 》 (et classés dans les catégories accidentels et indéterminés) lors de périodes de récession.

Ces observations générales suggèrent assurément l'existence de liens entre variables socio-économiques et la probabilité (ou les risques) d'incendies.

\section{PRÉSENTATION DES VARIABLES}

\subsection{Remarque sur le choix de la variable dépendante}

Au-delà de la question de la pertinence d'une approche socio-économique, voire strictement économique, aux fins d'expliquer la fréquence des incendies criminels, se pose la question du choix de la variable qui rendra compte de cette prévalence. En effet, Pettiway (1985) a montré que les coefficients sur les liens entre variables indépendantes et diverses formes de taux d'incendies changent de signification et même de signe en fonction des déterminants retenus.

À la base, afin d'éviter l'écueil consistant à « surestimer » le taux d'incidence de tout crime dans les quartiers centraux des villes, nous utilisons, tel que le propose Boggs (1965), un taux d'incidence dont le dénominateur reflète les opportunités des crimes étudiés plutôt qu'un taux basé sur la population. Le concept d'opportunité est ici fondamental. Ce concept étant adaptable selon que la recherche se concentre sur le délinquant ou l'événement, Davidson (1981) considère l' « opportunité » comme étant à la fois "prédisposante » (le milieu social, le tissu social et l'environnent physique du voisinage de l'acte criminel) et "précipitante ou actualisante " (les circonstances entourant l'acte).

Comme nous cherchons à mettre en lumière les opportunités dites prédisposantes de l'incendie criminel, nous utilisons un taux d'incendies criminels qui permette de tester le plus adéquatement possible l'effet de ces opportunités (relatives au bâti et à l'environnement socio-économique). Ainsi, la variable dépendante que nous utilisons dans cette étude est le nombre d'incendies par 10000 logements dans un secteur donné. 


\subsection{Présentation des variables}

Le tableau 2 ci-dessous présente les variables utilisées dans les différents modèles que nous développons. Il comprend des variables liées aux incendies et des variables socio-économiques. Bien que cela ne soit pas dit dans le tableau, comme pour les deux variables dépendantes INCENDIES-86 et INCENDIES-91, les variables indépendantes seront définies soit pour 1986 , soit pour 1991. Dans les tableaux qui présentent les résultats des modèles testés, nous représentons l'année pour laquelle la variable s'applique comme suit : TXCRIME-86 pour le taux de criminalité de 1986, TXCRIME-91 pour le taux de 1991, et ainsi de suite pour chacune des variables. Par ailleurs, certaines variables disponibles pour 1986 ne le sont pas pour 1991.

Tableau 2

Variables utilisées

Variables

Codes

Taux d'incendies criminels par 10000 logements

INCENDIES

Revenu moyen des ménages / Loyer mensuel moyen des logements

CAP

Charge hypothécaire mensuelle moyenne (Dépenses mensuelles moyennes des propriétaires) / Valeur moyenne des unités résidentielles

LEVIER

Proportion des ménages locataires dont le montant du loyer est supérieur

ou égal à $30 \%$ du revenu

Proportion de logements loués

LOC30PC

LOUES

Proportion de logements dont le loyer se chiffrait entre 0 et $200 \$$ par mois

LOY0A200

Proportion de logements dont la valeur allait de 150000 à $200000 \$$

LV150200K

Proportion de logements dont la valeur allait de 20000 à $50000 \$$

LV2050K

Proportion de logements construits avant 1946

PLC1946

Proportion de logements construits entre 1921 et 1945

PLC2145

Proportion de logements de 5 étages et plus

PLOG5ETA

Proportions de logements multiples

PLOGMULT

Proportion de ménages propriétaires dont les dépenses mensuelles

représentent $30 \%$ et plus du revenu

PROP30PC

Taux de chômage

TXCHOM

Taux de criminalité

TXCRIME

Liée à l'âge du bâti, la variable PLC1946 représente la proportion de logements dans le secteur construits avant 1946 alors que la variable PLC2145 représente la proportion des logernents du secteur construits entre 1921 et 1945. Étant donné que la très grande majorité des immeubles ont été construits après 1920 (en 1986, pour l'ensemble des secteurs de la ville, la proportion des logements construits avant 1945 s'élevait à $25,8 \%$ et $14,6 \%$ des logements dataient d'entre 1921 et 1945), en particulier dans les quartiers plus résidentiels, ces variables permettent de comparer l'effet du caractère plus 
ou moins récent des immeubles sur le taux d'incendies, et ce pour l'ensemble des immeubles ou presque. Nous pouvons nous attendre à ce que plus la proportion des logements âgés dans un secteur est grande, plus la probabilité d'incendies y soit élevée.

Liée aux types de bâtiments, la variable PLOG5ETA représente la proportion de logements du secteur qui ont 5 étages et plus alors que la variable PLOGMULT rend compte de la proportion d'unités résidentielles qui sont constituées de logements multiples. On relève dans la littérature sur l'incendie criminel que les immeubles de plus de 5 étages et à logements multiples sont plus enclins à subir des incendies criminels (l'étude de Bennett et al., 1987 suggère que la variable PLOGSETA influence le plus les taux d'incendies).

La variable LEVIER est le ratio de la charge hypothécaire mensuelle moyenne sur la valeur moyenne des unités résidentielles non attenantes dans chaque secteur de recensement. Les charges hypothécaires constituent souvent pour un ménage la plus grande part des dépenses et la source principale de leurs variations. Les coefficients associés à la variable LEVIER devraient être positifs : plus les propriétaires sont endettés, plus la probabilité qu'ils recourent à l'incendie criminel pour se dépêtrer d'une impasse financière est élevée. Telle que construite, cette variable traduit indirectement le ratio d'endettement des propriétaires d'immeubles (des charges mensuelles plus élevées relativement à la valeur des immeubles exprime un ratio d'endettement sur fonds propres plus élevés), d'où l'effet de LEviER lié à la propriété d'immeubles.

La variable CAP mesure le revenu moyen des ménages divisé par le loyer mensuel moyen. Elle traduit la capacité de paiement des occupants dans chacun des secteurs de la ville. À ce titre, la variable CAP est reliée aux variables LOC30P et PROP30PC. Comme le montrent Vallée et Clermont (1992), son coefficient pourra être instable au gré de la conjoncture économique. Dans un contexte de faible mobilité des occupants et de faible taux de vacance des logements, l'incitation des propriétaires à incendier leurs immeubles diminue avec la capacité des locataires de s'acquitter de leur loyer. Par contre, une forte capacité de paiement peut traduire l'impuissance des propriétaires à augmenter le loyer (soit en raison de contraintes institutionnelles, soit en raison d'une mobilité élevée des occupants alliée à un taux de vacance élevé des logements). Dans ce dernier cas, l'inclination à l'incendie criminel, et donc le taux d'incendies constatés, sera positivement corrélée à la capacité de payer des locataires.

La variable du taux de chômage, тхсном, mesure le taux de chômage dans chaque secteur. On estime que plus le taux de chômage est élevé dans un secteur donné, plus le taux d'incendies (donc la probabilité d'incendies 
criminels) y est est élevé. Les coefficients de la variable rendant compte de sa corrélation avec le taux d'incendies devraient être positifs. Par ailleurs, la variable TXCRIME représente le taux de criminalité défini pour chaque secteur comme le nombre d'effractions rapportées à la police divisé par le nombre de résidants de 15 ans ou plus. Cette variable incorpore l'effet des motifs d'incendie criminel autres que ceux à caractère économique. Le coefficient de TXCRIME devrait donc être positif. Dans les quartiers où la criminalité est plus élevée, les risques d'incendies criminels devraient être plus grands : on y recourt moins souvent à la justice pour régler les conflits et l'incendie criminel y est plus souvent utilisé pour camoufler d'autres crimes. Soulignons enfin que le statut de la victime (à savoir si l'incendie s'est déclaré chez un propriétaire ou un locataire) pourrait se révéler être un indice pertinent de la dynamique de l'ince-ndie criminel à but lucratif et qu'il aurait été bon de l'inclure dans l'étude. Malheureusement, la banque de données utilisée ne disposait pas d'un indicateur fiable de cette variable.

\section{PRÉSENTATION DES RÉSULTATS}

\subsection{Remarques d'ordre méthodologique}

La méthode des moindres carrés ordinaires appliquée sur les secteurs postaux est utilisée pour l'ensemble des tests. Pour toutes les régressions effectuées, la matrice variance-covariance des estimateurs a été vérifiée et l'on a corrigé l'hétéroscédasticité des erreurs lorsqu'on en a détecté la présence (test de White). L'échantillon contient environ 80 données, les fluctuations de sa taille étant dues à l'absence de données pour certaines variables indépendantes dans certains secteurs de Montréal, pour l'une ou l'autre des années choisies.

\subsection{Les modèles de base pour 1986 et 1991}

\subsubsection{Présentation du meilleur modèle de 1986 à partir des variables qui sont aussi disponibles pour l'année 1991}

Pour débuter, nous présentons, au tableau 3, la régression du taux d'incendies en 1986 avec les données de 1986, en nous limitant, à des fins de comparabilité, au groupe de variables pour lesquelles nous disposions aussi de données en 1991 (nous disposions de variables pour 1986 que nous n'avions pas pour 1991), de façon à pouvoir comparer les modèles optimaux des deux années à partir d'un stock de variables semblables et à pouvoir juger de la stabilité des déterminants du taux d'incendies criminels à Montréal entre 1986 et 1991 .

Le modèle pour l'année 1986 donne donc des résultats sensiblement en accord avec les attentes théoriques sur les déterminants des incendies 
criminels. Notons d'emblée que les variables LV2050K, LV150200K, et LOY0A200 sont absentes des tests (nous n'en disposions pas pour 1991). Par ailleurs, les variables PLC21-45 et PROP30PC ne sont pas significatives. La variable LEVIER semblerait capter l'effet de la variable PROP30PC en traduisant la pression financière pouvant peser sur les propriétaires dans un secteur donné.

Tableau 3

Taux d'incendies criminels par 10000 logements en 1986 avec les données de 1986 qui sont aussi disponibles en 1991 (2.1)

\begin{tabular}{lccc}
\hline & Coefficients & STAT-T & Prob $>|\mathrm{t}|$ \\
\hline CONSTANTE & $-17,97$ & $-3,47$ & 0,0008 \\
TXCHOM-86 & 78,21 & 3,68 & 0,0004 \\
LEVIER-86 & 1198,30 & 3,05 & 0,0030 \\
CAP-86 & 0,086 & 2,33 & 0,0221 \\
PLOG5ETA-86 & $\mathbf{5 , 6 1}$ & 1,80 & 0,0754 \\
\hline
\end{tabular}

$R^{2}$ ajusté $=0,28$

$F(4,83)=9,33$

* Le tableau 3, comme les tableaux suivants, montre les variables par ordre décroissant de signification, à l'exception de la constante, que nous présentons toujours en premier.

Le taux de chômage (тхсном) est la variable la plus significative. Ce résultat tend à confirmer que plus le taux de chômage est élevé dans un quartier, plus il y a de chances que soient provoqués des incendies de nature criminelle. Le coefficient de 78,21 signifie qu'une différence entre secteurs de $1 \%$ dans le taux de chômage se traduira, ceteris paribus, par un taux plus élevé d'incendies par 10000 logements de 0,782 , ce qui est assez élevé, vu la moyenne par secteur de 5,56 .

La variable LEVIER obtient un coefficient positif significatif. Cela tend à confirmer que des charges hypothécaires trop élevées peuvent amener certains propriétaires à incendier leurs immeubles (cf. Brady, 1983). La dimension du coefficient ne doit cependant pas nous tromper : la moyenne de la variable LEVIER est de 0,006 , de sorte qu'une hausse d'un millième de sa valeur amène une hausse de 1,20 dans le taux d'incendies sur 10000 logements.

La valeur positive significative du coefficient de CAP, variable traduisant l'aisance matérielle moyenne, indique que plus est grande la capacité de payer dans un quartier, plus grande est la propension à l'incendie criminel. Comme on l'a déjà dit, ce résultat contre-intuitif peut traduire l'impuissance des propriétaires à augmenter les loyers malgré une conjoncture économique favorable. Ainsi, une différence de 1 dans la valeur de CAP entre deux secteurs donnés se traduira par un taux de criminalité de 0,086 supérieur dans le secteur 
ayant la valeur de CAP la plus élevée, ce qui est une variation relativement marginale.

Enfin, le coefficient positif de la variable PLOG5ETA n'est significatif qu'à un niveau de signification de 7,5\%. Le coefficient de 5,61 signifie qu'une différence entre secteurs de $1 \%$ pour PLOG5ETA se traduit par un taux plus élevé de 0,056 incendies sur 10000 logements pour le secteur ayant la plus grande proportion de logements de 5 étages. Cette variation, quoique marginale, tendrait à confirmer que les logements plus grands sont plus fréquemment incendiés, comme le suggèrent plusieurs études.

\subsubsection{Le meilleur modèle pour 1991}

À partir du même ensemble de variables appliquées à 1991, le tableau 4 présente les paramètres de la meilleure régression de l'équation du taux d'incendies criminels de 1991 (avec les données de 1991). Les tableaux 3 et 4 révèlent des différences explicables soit en termes de changements structurels, soit en termes de cycle économique conjoncturel. Ces éléments font que des variables différentes ont le plus d'influence sur la spécification des taux d'incendies criminels.

\section{Tableau 4}

Taux d'incendies criminels en 1991

Tableau du meilleur modèle de 1991, avec les données de 1991 (3.1)

\begin{tabular}{lcrc}
\hline & Coefficients & STAT-T & Prob $>|\mathbf{t}|$ \\
\hline CONSTANTE & $-5,97$ & $-3,05$ & 0,003 \\
TXCHOM-91 & 58,42 & 3,24 & 0,002 \\
PLC1946-91 & 5,53 & 3,21 & 0,002 \\
LEVIER-91 & 937,50 & 3,13 & 0,002 \\
TXCRIME-91 & $-0,50$ & $-3,07$ & 0,003 \\
\hline
\end{tabular}

$R 2$ ajusté $=0,354$

$F(4,89)=13,21$

Le modèle pour 1991 comprend, à l'instar du modèle présenté au tableau 3, les variables LEVIER et TXCHOM, deux variables qui semblent stables entre les deux modèles. Cependant, les variables CAP et PLOG5ETA ne sont pas significatives (la constante est significative et négative).

Le taux de chômage тхсном a le coefficient le plus significatif et l'effet le plus élevé en termes absolus. Une variation de $1 \%$ du taux de chômage entre secteurs induit une variation de 0,58 incendies sur 10000 logements. Le coefficient positif de la variable PLC1946, dont la moyenne sur l'ensemble des secteurs en 1991 était de $21,8 \%$, suggère que les secteurs où les logements sont les plus âgés sont les plus susceptibles d'être le thêâtre d'incendies criminels. 
Le coefficient de la variable LEVIER (ratio des charges hypothécaires mensuelles moyennes sur la valeur moyenne des unités résidentielles) signifie quant à lui qu'une augmentation d'un millième de ce ratio augmente de près de 1 incendie sur 10000 logements le taux d'incendies criminels.

Le coefficient négatif significatif de TXCRIME peut surprendre : ceteris paribus, les secteurs postaux avec le plus bas taux de criminalité ont le plus haut taux d'incendies criminels par 10000 logements. L'explication la plus probable de ce résultat est liée à la conjoncture économique de 1991. Les incendies ont décidément augmenté lors de cette année (cf tableau 1) et les incendies criminels se sont répandus dans les secteurs à criminalité moins élevée, suggérant qu'en période de difficulté économique, la criminalité se répand tout en revêtant un caractère plus économique (fraude plutôt que vengeance ou pyromanie). Les incendies par vengeance ou pyromanie surviennent souvent dans des quartiers à criminalité élevée, alors que la fraude touche plus souvent les quartiers de propriétaires (les mieux nantis habitant les quartiers à criminalité plus faible). Ainsi, l'explication probable de ce résultat réside dans le rapport entre la théorie de la gentrification et la conjoncture économique : la pression financière sur les bien nantis qui ont dépassé leurs capacités de paiement les pousse à mettre le feu afin de frauder l'assurance.

\subsection{La robustesse des régressions}

Ayant pu constater, par les tableaux 3 et 4 , les différences entre variables significatives des meilleurs modèles « contemporains », nous nous attachons maintenant à vérifier la robustesse des modèles. Un aspect de celle-ci tient au pouvoir prédictif des données alors que l'autre aspect de la robustesse relève de la stabilité des variables et des paramètres des modèles dans le temps.

Tableau 5

Organisation des tests sur la robustesse des modèles

\begin{tabular}{lcccc}
\hline ROBUSTESSE & $\begin{array}{c}\text { Modète utilisé pour } \\
\text { les variables } \\
\text { indépendantes* }\end{array}$ & $\begin{array}{c}\text { Année des } \\
\text { données pour les } \\
\text { variables } \\
\text { indépendantes }\end{array}$ & $\begin{array}{c}\text { Année de la } \\
\text { variable } \\
\text { dépendante }\end{array}$ & Tableaux \\
(taux d'incendies) & \\
\hline & 1986 & 1986 & 1986 & Tableau 3 \\
Valeur prédictive & 1991 & 1991 & 1991 & Tableau 4 \\
des données & 1986 & 1986 & 1991 & Tableau 6 \\
Stabilité des modèles & 1991 & 1986 & 1991 & Tableau 7 \\
dans le temps & 1986 & 1991 & 1991 & Tableau 8 \\
\hline
\end{tabular}

* Note : le meilleur modèle pour 1986 est celui du tableau 3 ; le meilleur modèle de 1991, celui du tableau 4. 
Pour faire ressortir la robustesse du pouvoir prédictif des données, nous régressons les données de 1986 sur les taux d'incendies de 1991. Ainsi, à partir des données de 1986, pour ce qui est des variables indépendantes, et des données de 1991 pour ce qui est de la variable dépendante (c'est-à-dire les taux d'incendies par secteurs postaux), nous examinons les coefficients obtenus dans la régression des variables des modèles optimaux pour, respectivement, les années 1986 et 1991 (présentés dans les tableaux 3 et 4 respectivement). Cela permet de voir, à partir des données observées en 1986 , si pour expliquer les taux d'incendies cinq ans plus tard (en 1991), l'un ou l'autre modèle de régression explique mieux la somme des carrés des erreurs. Notre objectif ici est de vérifier si les données de 1986, utilisées dans un modèle approprié, auraient permis de prévoir les taux d'incendies de 1991 dans les secteurs de la ville de Montréal. Les tableaux 6 et 7 présentent les résultats de ces deux régressions.

En ce qui concerne l'aspect de la robustesse des régressions qui renvoie davantage à la stabilité des modèles dans le temps, nous utilisons dans les régressions, tant pour les variables indépendantes que les dépendantes, les données de la même année (soit 1986 ou 1991), en utilisant cependant, pour 1991, les variables du modèle optimal de 1986 (cf. tableau 3) et, pour 1986. les variables du modèle optimal de 1991 (cf. tableau 4). Cela nous permet de juger de la présence ou de l'absence de changements structurels dans les modèles, de voir en somme quelle est la signification, pour 1991, des coefficients des variables du meilleur modèle pour 1986 et vice-versa. Les résultats de ces régressions sont présentés respectivement dans les tableaux 8 et 9.

\subsubsection{Robustesse I : capacité prédictive}

\subsubsection{En utilisant le modèle de 1986}

Le tableau 6 reprend les variables du tableau 3. Il s'applique cependant aux taux d'incendies de 1991, avec le meilleur modèle obtenu à partir des données de 1986 (pour les variables disponibles pour 1991).

\section{Tableau 6}

Taux d'incendies criminels par 10000 logements en 1991 expliqués avec les données de 1986 avec le meilleur modèle de 1986

\begin{tabular}{lccc}
\hline & Coefficients & STAT-T & Prob $>|\mathbf{t}|$ \\
\hline CONSTANTE & $-7,10$ & $-2,14$ & 0,0351 \\
TXCHOM-86 & 47,97 & 3,48 & 0,0008 \\
LEVIER-86 & 715,48 & 2,98 & 0,0038 \\
PLOG5ETA-86 & 1,38 & 0,69 & 0,4934 \\
CAP-86 & 0,0157 & 0,66 & 0,5094 \\
\hline$R^{2}$ ajusté $=0,38$ & & & \\
$F(4,84)=13,74$ & & & \\
\hline
\end{tabular}


En raison de la forte « significativité » des coefficients des variables TXCHOM et LEVIER, le $\mathrm{R}^{2}$ du test, à 0,38 , est relativement élevé. Par ailleurs, alors que les variables CAP et PLOG5ETA de 1986 sont corrélées au taux d'incendies en 1986 (cf. tableau 3), elles ne restent pas significatives dans le temps et ne permettent donc pas de prédire le taux d'incendies en 1991. Quoi qu'il en soit, ce modèle parcimonieux offre un indice probant du caractère significatif des effets tardifs.

En effet, bien que l'effet absolu diminue de 1986 à 1991, il demeure que, ceteris paribus, plus le taux de chômage est élevé dans un secteur postal en 1986, plus le taux d'incendies y sera élevé en 1991. Au vu du coefficient de тхсном au tableau 4, une différence du taux de chômage entre secteurs de $1 \%$, en 1986, s'est traduite, 5 ans plus tard, par un taux différentiel d'incendies de 0,48 pour 10000 logements. En outre, l'effet de la variable LEVIER est aussi assez résistant dans le temps. Un coefficient de 715,48, au tableau 4, signifie qu'une variation, en 1986 de 0,001 de cette variable (entre secteurs) se sera traduite, en 1991, par une différence de 0,72 dans les taux d'incendies pour 10000 logements.

\subsubsection{En utilisant le meilleur modèle de 1991}

Dans cette démarche de vérification de la capacité prédictive des données à partir d'un modèle optimal (pour une année donnée), le tableau 7 montre les résultats de la régression des taux d'incendies de 1991 avec les variables du meilleur modèle pour 1991, mais sur la base des données de 1986. Le pendant de cette régression avec variables retardées se trouve donc au tableau 4.

\section{Tableau 7}

Taux d'incendies criminels de 1991 expliqués par les données de 1986 et le meilleur modèle de 199 ! (5.1)

\begin{tabular}{lccc}
\hline & Coefficients & t de Student & Prob $>|\mathbf{t}|$ \\
\hline CONSTANTE & $-3,63$ & $-1,96$ & 0,054 \\
TXCHOM-86 & 35,70 & 3,08 & 0,003 \\
TXCRIME-86 & $-0,31$ & $-2,94$ & 0,004 \\
PLC1946-86 & 3,01 & 2,00 & 0,049 \\
LEVIER-86 & 516,41 & 1,79 & 0,077 \\
\hline
\end{tabular}

$\mathrm{R}^{2}$ ajusté $=0,40$

$F(4,84)=15,00$

Les variables significatives de cette régression sont relativement robustes dans le temps. Le taux de chômage тхсном, le niveau des charges 
hypothécaires et la vétusté relative du bâtiment semblent de bonnes variables pour mettre à profit les données historiques en prévoyant, pour un secteur donné, les taux d'incendies qu'on y observera. Les coefficients de тхсном, TXCRIME et PLC 1946 sont toujours significatifs à un niveau de signification de $5 \%$, tandis que le coefficient de la variable LEvIER, relativement élevé en termes absolus, est significatif à un niveau de $10 \%$.

Ce modèle permet donc aussi d'envisager la possibilité d'élaborer des modèles prédictifs des taux d'incendies par secteurs urbains en utilisant des variables différées. Comme au tableau 4 , le taux de chômage demeure la variable dont le coefficient (positif) est le plus significatif et les coefficients de PLC1946 et TXCRIME demeurent respectivement positif et négatif, l'effet leur étant associé gardant le même signe, mais faiblissant avec le temps. Pour ces deux variables, la même interprétation peut être faite. Notons toutefois qu'avec TXCRIME, les secteurs à plus faible criminalité en 1986 (essentiellement les mêmes qu'en 1991) ont connu, ceteris paribus, des taux d'incendies proportionnellement plus élevés en 1991. En période de récession, la fraude à l'assurance augmenterait proportionnellement plus dans les secteurs urbains à taux de criminalité faible (souvent en processus de gentrification), où les propriétaires peuvent éprouver des difficultés d'ordre économique (cf. Therrien, Vallée et Dupuis, (1996).

\section{Robustesse II : stabilité des modèles}

\subsubsection{En utilisant le modèle de 1986 avec les données de 1991}

Le tableau 8 montre les résultats de la régression sur la base des variables du modèle obtenu pour l'année 1986 (présenté au tableau 3), mais en usant des données de 1991 et en appliquant la régression à la variable dépendante de 1991. Il s'agit ici de voir, sur le plan de la stabilité de la validité des modèles, si le meilleur modèle de 1986 est aussi valable lorsqu'il est appliqué aux taux d'incendies de 1991, avec les données de 1991.

Tableau 8

Taux d'incendies criminels par 10000 logements en 1991

Modèle utilisé pour l'année 1986, avec les données de 1991 (6.1)

\begin{tabular}{lccc}
\hline & $\begin{array}{c}\text { Paramètre } \\
\text { estimé }\end{array}$ & $\begin{array}{c}\mathbf{t} \text { de Student }\left(\mathbf{H}_{\mathbf{0}}\right. \\
\left(\mathbf{H}_{\mathbf{0}}=\mathbf{0}\right)\end{array}$ & Prob $>|\mathbf{t}|$ \\
\hline CONSTANTE & $-7,49$ & $-1,45$ & 0,1512 \\
LEVIER-91 & 1053,35 & 3,19 & 0,0020 \\
TXCHOM-91 & 76,76 & 2,80 & 0,0064 \\
PLOG5ETA-91 & $-2,37$ & $-1,23$ & 0,2217 \\
CAP-91 & 0,007 & 0,23 & 0,8153 \\
\hline
\end{tabular}

$R^{2}$ ajusté $=0,27$

$\mathrm{F}(4,85)=9,12$ 
Seules les variables LEVIER et TXCHOM demeurent significatives à un niveau de $5 \%$, prouvant une fois de plus leur robustesse et leur stabilité (entre modèles). Que ce soit en utilisant les données de 1986, comme au tableau 6, ou les données de 1991, comme nous le verrons au tableau 9, les variables PLOG5ETA et CAP, qui expliquent le taux d'incendies en 1986, ne sont pas des déterminants significatifs et donc de bons prédicteurs des taux d'incendies observés en 1991.

Considérant les variables liées aux caractéristiques des immeubles, c'est l'âge du parc d'immeubles (PLc1946) qui a de l'influence sur les taux d'incendies. Sur le plan des variables à caractère socio-économique, ce n'est plus la capacité de payer son loyer (CAP), mais le taux de criminalité (TXCRIM) dans le secteur qui est déterminant. Quels effets conjoncturels peuvent expliquer que les variables PLOG5ETA et CAP n'aient plus en 1991 de relation avec les taux d'incendies alors qu'elles en avaient en 1986 ? Concernant PLOG5ETA, peut-être qu'un certain seuil d'ancienneté, où les effets de l'âge prennent le pas sur ceux du nombre d'étages a été atteint entre 1986 et 1991. Concernant CAP, le problème en 1991 , période de creux dans le cycle économique, semble tenir davantage à la capacité des propriétaires de verser leurs paiements hypothécaires et de s'assurer de l'occupation minimale de leurs immeubles (effet capté par la variable LEVIER), qu'a la capacité des locataires de s'acquitter des loyers.

\subsubsection{En utilisant le modèle de 1991 avec les données de 1986}

Nous régressons ici le meilleur modèle de 1991 avec les données de 1986. L'idée est de tester, à partir d'un même ensemble de variables indépendantes, la stabilité du modèle optimal de 1991 appliqué à l'année 1986, de voir en somme dans quelle mesure le meilleur modèle de 1991 aurait été valable 5 ans plus tôt, en 1986.

Tableau 9

Taux d'incendies criminels par 10000 logements en 1986 selon le meilleur modèle obtenu pour 1991, avec les données de 1986

\begin{tabular}{lccc}
\hline & Coefficients & t de Student & Prob $>|\mathbf{t}|$ \\
\hline CONSTANTE & $-6,37$ & $-2,28$ & 0.025 \\
TXCHOM-86 & 49,76 & 3,22 & 0.002 \\
TXCRIME-86 & 0,75 & 2.82 & 0.006 \\
LEVIER-86 & 880,12 & 1,90 & 0.061 \\
PLC1946-86 & 0,29 & 0,12 & 0.90 \\
\hline
\end{tabular}

$R^{2}$ ajusté $=0,24$

$F(4,87)=4,63$ 
Le modèle du tableau 9 est le pendant de celui du tableau 4. Près du quart de la variance des erreurs est ici expliqué par les variables retenues. Par rapport à 1991, les coefficients des variables TXCHOM et TXCRIME restent clairement significatifs. Le coefficient de la variable LEVIER (toujours, à 880,12 , relativement élevé en termes absolu et relatif) demeure positif, mais n'est pas significatif à un niveau de $5 \%$. Celui de la variable PLC1946 n'est pas du tout significatif.

Le coefficient de TХCHOM est positif et demeure le plus grand en chiffres absolus. Par contre, contrairement au même modèle appliqué à 1991 (cf. tableau 4, où il est significativement négatif), le coefficient de TXCRIME est ici positif. En période de relative prospérité économique, conformément à nos attentes théoriques, les secteurs à plus forte criminalité montrent un taux d'incendies plus élevé, les incitatifs de la fraude à l'assurance étant absents chez les occupants ou propriétaires des quartiers aisés. La baisse de la significativité de la variable LEVIER rendrait aussi compte des motivations économiques changeantes, selon la conjoncture économique : les charges hypothécaires étant relativement moins fortes sur les propriétaires en 1986 qu'en 1991, leur lien avec l'incendie criminel est plus ténu. Dans le même ordre d'idées, le coefficient non significatif de PLC1946 peut rendre compte de deux facteurs concomitants : en 1986, la conjoncture étant favorable, le taux d'occupation relativement plus élevé ne permettait pas à l'âge des logements d'inciter les propriétaires à l'incendie, ni ne permettait au vandalisme dans les logements vacants de sévir. Tandis que le seuil atteint entre 1986 et 1991 expliquerait le caractère significatif du coefficient de PLC1946 pour 1991.

\section{CONCLUSIONS ET RECOMMANDATIONS}

\section{Constatations générales}

La présente recherche confirme l'hypothèse de liens statistiques entre le taux d'incendies, calculé sur le nombre de logements de divers secteurs urbains, et des variables socio-économiques non strictement liées aux caractéristiques des immeubles de ces secteurs. Des caractéristiques socioéconomiques peuvent donc être utilisées comme indicateurs de la probabilité qu'éclatent des incendies dans un secteur urbain donné.

Le taux de chômage (TXCHOM) et les charges hypothécaires (variable LEVIER) sont incontestablement liés au taux d'incendies, et ce quel que soit la phase du cycle économique. La comparaison des résultats pour les années 1986 et 1991 est à cet égard éloquente, ces deux années représentant clairement des années à l'opposé l'une de l'autre dans le cycle économique. Par ailleurs, des variables « intermédiaires » (à savoir les variables CAP, PLC1946, 
PLOG5ETA, TXCRIME) interviennent, mais leur présence et leur degré de signification dans les modèles ne sont pas constants dans toutes les régressions. À ce titre, nonobstant les effets de retard qu'on peut constater, les modèles sont dans l'ensemble relativement instables dans le temps, ce qui corrobore l'hypothèse de l'instabilité des motivations de l'incendiaire criminel dans le temps.

Ainsi, selon que l'on se situe au sommet ou au creux du cycle économique, des modèles différents s'appliquent, c'est-à-dire que des variables différentes seront les meilleurs indicateurs de la probabilité d'incendies dans des secteurs urbains donnés. D'une part, pour les facteurs socio-économiques, le signe des coefficients d'une même variable peut s'inverser (on l'a vu avec les variables CAP ou TXCHOM). D'autre part, les facteurs déterminant les taux d'incendies vont différer selon la période ou l'année considérée. On est donc face à une double instabilité, géographique et temporelle, qui rendrait, à la base, toute tentative d'élaboration d'un modèle de prédiction valable très compliquée.

\section{Discussion des problèmes liés à l'approche décrite dans l'étude}

L'indisponibilité des données nécessaires à l'analyse pour l'époque où l'on veut faire une prévision sur des taux d'incendies, pose problème. Les modèles développés ne sont calculés qu'a posteriori. L'instabilité dans le temps des modèles optimaux est également problématique, dans la mesure où ils changent au gré de la conjoncture. Il faudrait pouvoir disposer d'un modèle économétrique préliminaire qui indique quelle sera la conjoncture la plus probable dans, par exemple, cinq ans. À partir de cette prédiction, on pourrait alors appliquer le modèle de prévision pertinent.

Enfin, ces difficultés « techniques »sont aggravées par le fait que les données servant à faire les tests sont celles des secteurs de recensement, disponibles une fois tous les cinq ans, et ce après un délai non négligeable. Quoi qu'il en soit, les modèles développés sont, à tout le moins, fort utiles pour établir quels sont les paramètres à considérer dans les polices d'assurance, surtout au stade de la tarification. De plus, ils sont un indice clair qu'à l'échelle des secteurs urbains, les phénomènes agrégés jouent un rôle.

\section{Facteurs pris en compte par les compagnies d'assurances et nécessité d'une documentation plus détaillée sur l'assuré et ses locataires}

Bien que le BAC ne fasse pas de recommandation officielle sur la façon de tarifer, laissant le marché discriminer les couvertures non concurrentielles, il ressort que les compagnies d'assurances devraient systématiquement tenir compte du contexte socio-économique dans lequel s'inscrit le risque assuré et non pas seulement de son cadre physique. Et ce même si ce dernier, il est vrai, entretient souvent une forte corrélation avec le précédent. 
Ainsi, en fonction des paramètres mis au jour, si on apprend que le chômage, dans le quartier de l'assuré, ou que les charges hypothécaires moyennes du propriétaire en proportion de son revenu ont augmenté, on peut plus pertinemment établir le prix des assurances (primes et franchises) à payer. À cet égard, il a été prouvé qu'un suivi plus marqué de la situation des assurés réduit de $50 \%$ les taux d'incendies déclarés par ceux-ci. On peut penser ici à un système organisé permettant que les assurés, lorsque leur situation se modifie défavorablement, soient informés de l'attention spéciale portée à leur dossier. Cela peut réduire sensiblement le risque moral, de même que l'incitation à régler ses problèmes financiers par une fraude à l'assurance. La prise en compte de la dimension socio-économique des incendies ainsi que la mise « sous surveillance » d'assurés qui en sont informés sont des pistes d'action que les assureurs ne peuvent ni ignorer, ni négliger.

Nous proposons donc que les assureurs accordent une attention particulière aux demandes de souscription d'assurance des résidents des quartiers les plus à risque. Il serait pertinent de fonder empiriquement ce risque en élaborant, spécifiquement pour Montréal, un indice de risque d'incendie à partir de variables révélatrices du phénomène. Nous en avons identifié quelques-unes dans cette étude, mais nous croyons que de plus amples efforts ne seraient pas vains. Un tel instrument pourrait permettre d'évaluer assez précisément le niveau de risque associé à un secteur. Idéalement, on pourrait en arriver à prédire, à un niveau de certitude satisfaisant, les risques d'incendies qui y sont corrélés. Une plus grande surveillance devrait ainsi être exercée dans les zones présentant des profils à risque de fraude plus élevée ainsi que des caractéristiques socio-économiques et financières particulières. La réduction de l'incidence des feux et l'amélioration de la capacité des assureurs à calculer les probabilités, et donc à prédire les pertes dues aux incendies, pourrait réduire significativement les réclamations et, par conséquent, les primes.

Sur le modèle du Early Warning System et de l'APR (Arson Pattern Recognition) américains qui s'efforcent de détecter les bâtiments sujets à incendie frauduleux ou à détérioration (des systèmes d'aide à l'enquête préventive lors de la souscription), on peut imaginer la constitution d'un système de prévision économétrique assez précis qui permettrait aux compagnies d'assurances de discriminer, en fonction des variables socioéconomiques du secteur concerné, les quartiers de Montréal les plus à risque et, en conséquence, de fixer le montant des primes non plus seulement en fonction des caractéristiques des immeubles. Nous espérons que ce travail aura contribué à l'élaboration d'un tel modèle de prédiction des taux d'incendies. 


\section{BIBLIOGRAPHIE}

BENNETT, W. D., MERLO A. V., ET LEIKER K.K. (1987), " Geographical Patterns of Incendiary and Accidental Fires in Springfield, Massachusetts, 1980-1984 », Journal of Quantitative Criminology. vol. 3, $\mathrm{n}^{\circ} \mathrm{1}, 1987$.

BOGGS, S. L. (1965), «Urban Crime Patterns », American Sociological Review, vol. 30, pp. 899-908.

BRADY, J. P. (1983), «Arson, Urban Economy, and Organized Crime : The Case of Boston », Social Problems, vol. 31, n' 1, octobre.

CLERMONT, Y. (1990), Un modèle économétrique de la configuration spatiale de l'incendie criminel de bâtiments résidentiels à Montréal : un lien avec l'état du stock immobilier résidentiel, mémoire de maîtrise, École des Hautes Études Commerciales, décembre.

CLERMONT, Y. ET VALLÉE, L. (1992), « L'instabilité des motivations de l'incendie criminel dans le secteur résidentiel à Montréal », L'Actualité économique, Montréal, septembre.

CLONIGER, D. A. (1978) « Risk and Abandonment », Joumal of Risk and Insurance. pp. 494-504.

DAVIDSON, R. N. (1981) Crime and Entironment, London, Croom Helm.

GÉNÉREUX, P. ET LAMOUREUX, P. (1987), Document statistique sur les incendies criminels sur le territoire de la communauté urbaine de Montréal, Société de police de la Communauté urbaine de Montréal, rapport interne inédit, $16 \mathrm{p}$.

HERSHBARGER. R. A. ET MILLER, R. K. (1978), « The Impact of Economic Conditions on the Incidence of Arson ". Joumal of Risk and Insurance, vol. XLV, $\mathrm{n}^{\circ} 2$, juin.

MÜNCHENER RÜCKVERSICHERUNGS-GESELLSCHAFT (1984), L'Incendie volontaire, $56 \mathrm{p}$.

PETTIWAY, LEON E. (1989), « Measures of Opportunity and the Calculation of the Arson Rate: The Connection Between Operationalization And Association», Journal of Quantitative Criminology, vol. 1, n³ 3, 1985, pp. 241-268.

THERRIEN. F., VALLÉE, L.. DUPUIS. S. (1996), La gentrification et l'incendie criminel dans trois quartiers de Montréal, Rev'ue Assurances, $n^{\circ}$ 1, avril. pp. 83-140. 